\title{
VERTEX PARTITIONS OF CHORDAL GRAPHS
}

\author{
DAVID R. WOOD
}

\begin{abstract}
A $k$-tree is a chordal graph with no $(k+2)$-clique. An $\ell$-treepartition of a graph $G$ is a vertex partition of $G$ into 'bags', such that contracting each bag to a single vertex gives an $\ell$-tree (after deleting loops and replacing parallel edges by a single edge). We prove that for all $k \geq \ell \geq 0$, every $k$-tree has an $\ell$-tree-partition in which every bag induces a connected $\lfloor k /(\ell+1)\rfloor$-tree. An analogous result is proved for oriented $k$-trees.
\end{abstract}

\section{INTRODUCTION}

Let $G$ be an (undirected, simple, finite) graph with vertex set $V(G)$ and edge set $E(G)$. The neighbourhood of a vertex $v$ of $G$ is denoted by $N(v)=\{w \in V(G)$ : $v w \in E(G)\}$. A chord of a cycle $C$ is an edge not in $C$ whose endpoints are both in $C$. $G$ is chordal if every cycle on at least four vertices has a chord. A $k$-clique $(k \geq 0)$ is a set of $k$ pairwise adjacent vertices. A $k$-tree is a chordal graph with no $(k+2)$-clique. The tree-width of $G$, denoted by $\operatorname{tw}(G)$, is the minimum $k$ such that $G$ is a subgraph of a $k$-tree. It is well known that $G$ is a $k$-tree if and only if $V(G)=\emptyset$, or $G$ has a vertex $v$ such that $G \backslash v$ is a $k$-tree, and $N(v)$ is a $k^{\prime}$-clique for some $k^{\prime} \leq k$.

Let $G$ and $H$ be graphs. The elements of $V(H)$ are called nodes. Let $\left\{H_{x} \subseteq\right.$ $V(G): x \in V(H)\}$ be a set of subsets of $V(G)$ indexed by the nodes of $H$. Each set $H_{x}$ is called a bag. The pair $\left(H,\left\{H_{x} \subseteq V(G): x \in V(H)\right\}\right)$ is an $H$-partition of $G$ if:

- $\forall$ vertices $v$ of $G, \exists$ node $x$ of $H$ with $v \in H_{x}$, and

- $\forall$ distinct nodes $x$ and $y$ of $H, H_{x} \cap H_{y}=\emptyset$, and

- $\forall$ edge $v w$ of $G$, either

- $\exists$ node $x$ of $H$ with $v \in H_{x}$ and $w \in H_{x}$, or

- $\exists$ edge $x y$ of $H$ with $v \in H_{x}$ and $w \in H_{y}$.

For brevity we say $H$ is a partition of $G$. A $k$-tree-partition is an $H$-partition for some $k$-tree $H$. A tree-partition is a 1-tree-partition. Tree-partitions were independently introduced by Seese [13] and Halin [12], and have since been investigated

Date: October 22, 2018.

1991 Mathematics Subject Classification. 05C15 (Coloring of graphs and hypergraphs).

Key words and phrases. graph, chordal graph, $k$-tree, vertex partition, $H$-partition, treepartition, tree-width.

Research supported by NSERC and COMBSTRU. 
by a number of authors $2,3,6,6,12,13$. The main property of tree-partitions that has been studied is the maximum cardinality of a bag, called the width of the tree-partition. The minimum width over all tree-partitions of a graph $G$ is the tree-partition-width ${ }^{1}$ of $G$, denoted by $\operatorname{tpw}(G)$. A graph with bounded degree has bounded tree-partition-width if and only if it has bounded tree-width 7]. In particular, for every graph $G$, Seese 13. proved that $\operatorname{tw}(G) \leq 2 \operatorname{tpw}(G)-1$, and Ding and Oporowski [6] proved that $\operatorname{tpw}(G) \leq 24 \operatorname{tw}(G) \max \{\Delta(G), 1\}$, where $\Delta(G)$ is the maximum degree of $G$. See [1, 5, 8, 9] for other results related to tree-width and vertex partitions.

Tree-partition-width is not bounded above by any function solely of tree-width. For example, wheel graphs have bounded tree-width and unbounded tree-partitionwidth, as observed by Bodlaender and Engelfriet [3]. Thus, it seems unavoidable that the maximum degree appears in an upper bound on the tree-partition-width. This fact, along with other applications, motivated Dujmović et al. 10, 11] to study the structure of the bags in a tree-partition. In this paper we continue this approach, and prove the following result (in Section 2).

Theorem 1. Let $k$ and $\ell$ be integers with $k \geq \ell \geq 0$. Let $t=\lfloor k /(\ell+1)\rfloor$. Every $k$-tree $G$ has an $\ell$-tree-partition in which each bag induces a connected $t$-tree in $G$.

It is easily seen that Theorem 1 is tight for $G=K_{k+1}$ and for all $\ell$. Note that Theorem 1 can be interpreted as a statement about chromomorphisms (see 15, 16]).

Dujmović et al. 10, 11] proved that every $k$-tree has a tree-partition in which each bag induces a $(k-1)$-tree. Thus Theorem 1 with $\ell=1$ improves this result. That said, the tree-partition of Dujmović et al. 10, 11] has a number of additional properties that were important for the intended application. We generalise these additional properties in Section 3 The price paid is that each bag may now induce a $(k-\ell)$-tree, thus matching the result of Dujmović et al. 10, 11] for $\ell=1$. Note that the proof of Dujmović et al. 10, 11] uses a different construction to the one given here.

\section{Proof of Theorem 1}

We proceed by induction on $|V(G)|$. If $V(G)=\emptyset$, then the result holds with $V(H)=\emptyset$ regardless of $k$ and $\ell$. Now suppose that $|V(G)| \geq 1$. Thus $G$ has a vertex $v$ such that $G \backslash v$ is a $k$-tree, and $N(v)$ is a $k^{\prime}$-clique for some $k^{\prime} \leq k$. By induction, $G \backslash v$ has an $\ell$-tree-partition $H$ in which each bag induces a connected $t$-tree. Let $C=\left\{x \in V(H): N(v) \cap H_{x} \neq \emptyset\right\}$. Since $N(v)$ is a clique, $C$ is a clique of $H$ (by the definition of $H$-partition). Since $H$ is an $\ell$-tree, $|C| \leq \ell+1$.

Case 1. $|C| \leq \ell$ : Add one new node $y$ to $H$ adjacent to each node $x \in C$. Since $C$ is a clique of $H$ and $|C| \leq \ell, H$ remains an $\ell$-tree. Let $H_{y}=\{v\}$. The other

\footnotetext{
${ }^{1}$ Tree-partition-width has also been called strong tree-width [3, 13].
} 
bags remain unchanged. Since $t \geq 0, H_{y}$ induces a connected $t$-tree $\left(=K_{1}\right)$ in $G$. Thus $H$ is now a partition of $G$ in which each bag induces a connected $t$-tree in $G$.

Case 2. $|C|=\ell+1$ : There is a node $y \in C$ such that $\left|N(v) \cap H_{y}\right| \leq t$, as otherwise $|N(v)| \geq(t+1)|C|=(\lfloor k /(\ell+1)\rfloor+1)(\ell+1) \geq k+1$. Add $v$ to the bag $H_{y}$. Let $u \in N(v) \cap H_{y}$. Every neighbour of $v$ not in $H_{y}$ is adjacent to $u$ (in $G \backslash v$ ). Thus $H$ is a partition of $G$. $H_{y}$ induces a connected $t$-tree in $G$, since $H_{y} \backslash\{v\}$ induces a connected $t$-tree in $G \backslash v$, and the neighbourhood of $v$ in $H_{y}$ is a clique of at least one and at most $t$ vertices. The other bags do not change. Thus each bag of $H$ induces a connected $t$-tree in $G$.

\section{Oriented Partitions}

Let $G$ be an oriented graph with arc set $A(G)$. Let $\widehat{G}$ be the underlying undirected graph of $G$. The in- and out-neighbourhoods of a vertex $v$ of $G$ are respectively denoted by $N^{-}(v)=\{u \in V(G): u v \in A(G)\}$ and $N^{+}(v)=\{w \in V(G)$ : $v w \in A(G)\}$. It is easily seen that an (undirected) graph $G$ is a $k$-tree if and only if there is an acyclic orientation of $G$ such that for every vertex $v$ of $G, N^{-}(v)$ is a $k^{\prime}$-clique for some $k^{\prime} \leq k$. An oriented graph with this property is called an oriented $k$-tree. Let $G$ and $H$ be oriented graphs. An oriented $H$-partition of $G$ is

an $\widehat{H}$-partition of $\widehat{G}$ such that for every arc $x y$ of $H$, and for every edge $v w$ of $\widehat{G}$ with $v \in H_{x}$ and $w \in H_{y}, v w$ is oriented from $v$ to $w$. This concept is similar to an oriented homomorphism (see [4, 14] for example).

Theorem 2. Let $k$ and $\ell$ be integers with $k \geq \ell \geq 0$. Let $t=k-\ell$. Every oriented $k$-tree $G$ has an oriented $\ell$-tree partition $H$ in which each bag induces a weakly connected oriented $t$-tree in $G$. Moreover, for every node $x$ of $H$, the set of vertices $Q(x)=\bigcup_{v \in H_{x}}\left(N^{-}(v) \backslash H_{x}\right)$ is a $k^{\prime}$-clique of $G$ for some $k^{\prime} \leq k$.

The construction in the proof of Theorem 2 only differs from that of Theorem 1 in the choice of the node $y$ in Case 2 .

Proof. We proceed by induction on $|V(G)|$. If $V(G)=\emptyset$, then the result holds with $V(H)=\emptyset$ regardless of $k$ and $\ell$. Now suppose that $|V(G)| \geq 1$. Since $G$ is acyclic, there is a vertex $v$ of $G$ such that $N^{+}(v)=\emptyset, N^{-}(v)$ is a $k^{\prime}$-clique for some $k^{\prime} \leq k$, and $G \backslash v$ is an oriented $k$-tree. By induction, there is an oriented $\ell$-tree-partition $H$ of $G \backslash v$ in which each bag induces a weakly connected oriented $t$-tree in $G \backslash v$. Moreover, for every node $x$ of $H, Q(x)$ is a $k^{\prime}$-clique for some $k^{\prime} \leq k$. Let $C=\left\{x \in V(H): N^{-}(v) \cap H_{x} \neq \emptyset\right\}$. Since $N^{-}(v)$ is a clique, $C$ is a clique of $H$. Since $H$ is an oriented $\ell$-tree, $|C| \leq \ell+1$.

Case 1. $|C| \leq \ell$ : Add one new node $y$ to $H$ adjacent to each node $x \in C$. Orient each new edge from $x$ to $y$. Obviously $H$ remains acyclic. Since $C$ is a clique of $H$ and $|C| \leq \ell, H$ remains an oriented $\ell$-tree. Let $H_{y}=\{v\}$. The other bags are unchanged. Since $t \geq 0, H_{y}$ induces a weakly connected oriented $t$-tree $\left(=K_{1}\right)$ in $G$. All edges of $G$ that are incident to a vertex in $H_{y}$ are oriented into the vertex in 
$H_{y}$. Thus $H$ is now an oriented partition of $G$ in which each bag induces a weakly connected oriented $t$-tree in $G$. Now $Q(y)=N^{-}(v)$, which is a $k^{\prime}$-clique for some $k^{\prime} \leq k . Q(x)$ is unchanged for nodes $x \neq y$. Hence the theorem is satisfied.

Case 2. $|C|=\ell+1$ : The clique $C$ induces an acyclic tournament in $H$. Let $y$ be the sink of this tournament. Since $\left|N^{-}(v) \cap H_{x}\right| \geq 1$ for every node $x \in C \backslash\{y\}$, $\left|N^{-}(v) \cap H_{y}\right| \leq k^{\prime}-(|C|-1) \leq k-\ell=t$. Add $v$ to the bag $H_{y}$.

Consider a neighbour $u$ of $v$. Since $N^{+}(v)=\emptyset$, $u v$ is oriented from $u$ to $v$. Say $u \in H_{z}$ with $z \neq y$. Then $z$ is in the clique $C$. Thus $z y$ is an edge of $H$. Since $y$ is a sink of $C, z y$ is oriented from $z$ to $y$. Thus $H$ is now an oriented partition of $G$. $H_{y}$ induces a weakly connected oriented $t$-tree in $G$, since $H_{y} \backslash\{v\}$ induces an oriented $t$-tree in $G \backslash v$, and the in-neighbourhood of $v$ in $H_{y}$ is a clique of at least one and at most $t$ vertices. The other bags do not change. Thus each bag of $H$ induces a weakly connected oriented $t$-tree in $G$.

$Q(y)$ is not changed by the addition of $v$ to $H_{y}$, as there is at least one vertex $u \in N^{-}(v) \cap H_{y}$, and any vertex in $N^{-}(v) \backslash H_{y}$ is also in $N^{-}(u) \backslash H_{y}$. For nodes $x \neq y, Q(x)$ is unchanged by the addition of $v$ to $H_{y}$, since $v$ is not in the inneighbourhood of any vertex. Hence the theorem is satisfied.

\section{ACKNOWLEDGEMENTS}

Thanks to Matthew DeVos, Vida Dujmović, Attila Pór, and Ricardo Strausz for stimulating discussions.

\section{REFERENCES}

[1] N. Alon, G. Ding, B. Oporowski, and D. Vertigan, Partitioning into graphs with only small components. J. Combin. Theory Ser. B, 87(2):231-243, 2003.

[2] H. L. Bodlaender, A note on domino treewidth. Discrete Math. Theor. Comput. Sci., 3(4):141-150, 1999.

[3] H. L. Bodlaender and J. Engelfriet, Domino treewidth. J. Algorithms, 24(1):94-123, 1997.

[4] O. V. Borodin, D. Fon-Der-Flaass, A. V. Kostochka, A. Raspaud, And E. Sopena, On deeply critical oriented graphs. J. Combin. Theory Ser. $B, \mathbf{8 1 ( 1 ) : 1 5 0 - 1 5 5 , ~} 2001$.

[5] M. DeVos, G. Ding, B. Oporowski, D. P. Sanders, B. Reed, P. SeyMOUR, AND D. Vertigan, Excluding any graph as a minor allows a low tree-width 2-coloring. J. Combin. Theory Ser. B, 91(1):25-41, 2004.

[6] G. Ding And B. Oporowski, Some results on tree decomposition of graphs. J. Graph Theory, 20(4):481-499, 1995.

[7] G. Ding and B. Oporowski, On tree-partitions of graphs. Discrete Math., 149(1-3):45-58, 1996. 
[8] G. Ding, B. Oporowski, D. P. Sanders, and D. Vertigan, Partitioning graphs of bounded tree-width. Combinatorica, 18(1):1-12, 1998.

[9] G. Ding, B. Oporowski, D. P. Sanders, and D. Vertigan, Surfaces, tree-width, clique-minors, and partitions. J. Combin. Theory Ser. B, 79(2):221-246, 2000.

[10] V. Dujmović, P. Morin, And D. R. Wood, Layout of graphs with bounded tree-width. SIAM J. Comput., to appear.

[11] V. Dujmović And D. R. Wood, Tree-partitions of $k$-trees with applications in graph layout. In H. Bodlaender, ed., Proc. 29th Workshop on Graph Theoretic Concepts in Computer Science (WG'03), vol. 2880 of Lecture Notes in Comput. Sci., pp. 205-217, Springer, 2003.

[12] R. Halin, Tree-partitions of infinite graphs. Discrete Math., 97:203-217, 1991.

[13] D. Seese, Tree-partite graphs and the complexity of algorithms. In L. BuDACH, ed., Proc. International Conf. on Fundamentals of Computation Theory, vol. 199 of Lecture Notes in Comput. Sci., pp. 412-421, Springer, 1985.

[14] E. Sopena, The chromatic number of oriented graphs. J. Graph Theory, 25(3):191-205, 1997.

[15] R. Strausz, On Separoids. Ph.D. thesis, Universidad Nacional Autónoma de México, México, 2003.

[16] R. Strausz, Representation of separoids and a Tverberg-type problem. In M. Mareš, ed., Proc. 9th Midsummer Combinatorial Workshop, vol. 686 of KAM-DIMATIA Series, pp. 42-51, Department of Applied Mathematics, Charles University, Prague, Czech Republic, 2004.

School of Computer Science, Carleton University, Ottawa, Canada. Department of Applied Mathematics, Charles University, Prague, Czech Republic.

E-mail address: davidw@scs.carleton.ca 